\title{
Zur Technik der Pleura- und Lungennaht.
}

\author{
Von Prof. Dr. Poenaru-Caplescu, Bukarest.
}

Bei Lungenwunden halte ich den Eingriff für unbedingt notwendig in den Fällen, wo die Blutung eine starke und der Zustand des Kranken ein schwerer ist.

Diesen Zustand der Schwere können wir bei einiger Erfahrung in dieser Art von Verletzungen sogar ziemlich genau beurteilen.

Auch in den sehr schweren Fällen können wir die Kranken nicht hilflos sterben lassen. Einer meiner Kranken war in extremis. Nur durch endovenöse Injektion von $2000 \mathrm{~g}$ physiologischer Kochsalzlösung habe ich die Operation glücklich bewerkstelligen können. Einer der Assistenten glaubte wegen des synkopalen Zustandes, in dem sich der Verletzte befand, daß alles vergeblich sein würde, und zögerte, eine Hilfeleistung zu versuchen. Una der Kranke kam ohne Zwischenfall zur Heilung.

Bei meinen Fällen bin ich nie einem unangenehmen Emphysem oder einer Lungenhernie, die eine Indikation zum Eingriff gewesen wäre, begegnet. Allein die Blutung gab bei den 6 von mir operierten Fällen die formelle Indikation zum Eingriff $a b$.

Dic Infektion, die meiner Ansicht nach häufig auf den Eingriff zurückzuführen ist, erfordert eine rigoröse Asepsis. Die Antisepsis, die unglücklicherweise noch in gro$\mathrm{Bem} \mathrm{MaBstabevorgenommen} \mathrm{wird,} \mathrm{ist} \mathrm{für} \mathrm{die} \mathrm{citrigen}$ Pleuritiden und Septikämien nach dem Eingriff verantwortlich $z u$ machen.

Wegen der $A$ tisepsis wird die Asepsis nicht streng durchgeführt.

Was mich betrifft, so habe ich, seit ich $\Lambda$ ssistent von Jonnescu bin, die Antiseptica vollständig aufgegeben. Seit mehr als 13 Jahren mache ich von Sublimat, Karbolsäure, Jodoform oder anderen Antisepticis keinen Gebrauch mehr. 
Nur Jodtinktur verwendete ich für die Asepsis des Operationsfeldes bei den beiden letzten Fällen von Lungenwunden, die ich im letzten Sommer dringlich bei Nacht operierte.

Liegen die Lungenwunden ganz in der Nähe des Hilus, so glaube ich, daß sie sich dennoch vernähen lassen. Nur in einem Fall (dem sechsten und letzten von mir operierten) fand ich eine zerfetzte Lungenwunde infolge der ausgedehnten Pleuraverwachsungen, auf die genau die Beschreibung $\mathrm{B}$ a udets paßte. Ich war gezwungen zu tamponieren; die Bronchienäste waren geöffnet. An den folgenden Tagen flo $B$ durch die tamponierte Wunde eine scröse, mit schleimig-eitriger Bronchialsekretion untermischte Flüssigkeit ab. Der Kranke genas ohne Zwischenfall.

Grunert, Holz, Souligoux, Martel und vor allem $B$ a u det und $Z$ eidler haben vielleicht recht, Interventionisten zu sein.

Wir müssen uns immer vor Augen halten, daß diese Thoraxwunden recht häufig thorako-abdomina le sein können (zwei von meinen sechs Fällen) und daß der Fingriff in diesen Fällen formell indiziert ist.

Ich habe 12 Kranke wegen vermutlicher Lungenverletzungen (bei 2 Fällen dachte ich sogar an die Möglichkeit einer Herzwunde) außer I (geheilten) Fall von Herzwunde und 4 Fällen von thorako-abdominalen Wunden ohne Lungenverletzung in Behandlung gehabt (nur ein Kranker kam zum Iixitus: Leberwunde mit manifester Peritonitis bei der Operation).

Unter diesen 12 Fällen von Lungenwunden, wovon 2 lälle mit Verletzungen der Leber und des Zwerchfelles, fand ich eine formelle Indikation nur bei 6 Fällen und der Eingriff erzielte die Heilung der Kranken. Von diesen 6 Fällen konnten, wenn sie nicht operiert worden wären, wenigstens zwei an der Leberverletzung sterben.

I)ic: übrigen 6 Fälle von mittlerer Schwere mit Hämoptysis und Hämothorax kamen ohne Operation zur Heilung.

Ich habealso $12 \mathrm{Kranke}$ it Lungenverletzun. gen und I 2 Heilungen.

Was die Drainage der Pleura betrifft, so habe ich sie ats einer der ersten seit meiner Arbeit über die thorako-abdominalen 
Verletzungen ${ }^{1}$ ) bekämpft. Ich war gegen die Drainage des P'erikards und der Pleura im Jahre 1907 gelegentlich eines Falles von Herznaht. Mein Kranker, der einen Messerstich im linken Ventrikel hatte, genas vollkommen ohne jeglichen Zwischenfall. obwohl ich sowohl Perikard wie Pleura ohne Drainage verschloß ${ }^{2}$ ).

In meiner Arbeit über die thorako-abdominalen Verletzungen behauptete ich, daß die Drainage nur in den Fällen notwendig ist, wo der Eingriff spät erfolgt oder im Augenblick des Eingriffes bereits Infektionserscheinungen der Pleura bestehen.

Da ich in den Jahren 1907, 1908, 1909 Gelegenheit gehabt hatte, 4 Fälle von Lungenwunden (in der Jonnescuschen Abteilung) zu operieren, veröffentlichte ich im Anschluß daran eine Arbeit über die Technik der dringlichen Lungennaht.

In dieser 39 Seiten starken Arbeit ${ }^{3}$ ) mit einem möglichst voliständigen Literaturverzeichnis schrieb ich: „Die Drainage der Pleurahöhle ist nur in den Fällen indiziert, wo der Eingriff bei infizierter Wunde spät erfolgt; bei den dringlichen Eingriffen sind wir der Ansicht, daB die Drainage nicht nur unnütz, sondern sogar gefährlich ist, da sie 7. uweilen tödliche Sekundärinfektionen der Pleura begünstigen kann.

Im Mai 1907 (Revista de chirurgie p. 273, Diskussion in der chirurgischen Gesellschaft zu Bukarest) hatte ich diesen Punkt weiter betont.

Was den chirurgischen Pneumothorax betrifft, so habe ich mich bei meinen 17 Fällen von Pleurachirurgie nie zu beklagen gehabt; nie hatte ich unangenehme Zufälle zu verzeichnen.

Weiterhin hatten mir die Igo6 mit Dobrinescu und 1907 mit $\mathrm{M}$ it rea unternommenen Versuche (thorako-abdominale Wun den bei der ersten Serie von iz Hunden und Lungenwunden

1) Poe n a r u - C a p l e s c u, Contributiuni la studiul chirurgiei plàgilor toraco-abdominale. (Revista de chirurgie 1906, 26 Seiten.)

2) -, Plagă penetranta a toracelui, a pericardului si a ventriculului stàng cardioraphie, vindecare. (Spitalul 1907, I Abb.)

3) - - Technica suturilor pulmonare de urgentă. (Revista de chirurgie I909, II Abb.) 
bei der zweiten Serie von I 2 Hunden) die Unschädlichkeit des allmählich erzeugten Pneumothorax gezeigt. Die Resultate sind in zwei Bukarester Dissertationen niedergelegt.

Die besonderen Apparatesind somitzum Eingriff ander Lunge oder am Herzen nicht notwendig. Außerdem sind sie kostspielig, recht kompliziert und nicht in allen Krankenhäusern zu haben.

Aspiration der Pleuraluft, Thorakozentese und Pleurotomie werden wertvolle Hilfsmittel bei der Nachbehandlung scin.

Die Dimensionen des Thoraxlappens müssen groß genug sein, um im Notfalle die Einführung der ganzen Hand zu gestatten, wodurch die Naht und die vollständige Absuchung der Lunge und der Wände der Pleurahöhle enorm erleichtert werden wird.

Der Lappen mit innerem Scharnier verdient allemal dann den Vorzug, wenn die Wunde beim Sternum liegt. Auf diesc Weise erfolgt die Mobilisation des Lappens durch die sternokostalen Knorpel rascher, was bei gewissen Eingriffen von großer Wichtigkeit ist.

In diesen Fällen ist die Mammaria interna zu überwachen, dic gleich den Intercostales verletzt sein könnte.

Die Naht der Lunge hat stets mit Katgut zu geschehen (Seide wäre aufzugeben). Ich habe stets Katgut Nr.o von Leclerc und Triollet verwendet.

Dic Knopfnähte gehen $1 \mathrm{~cm}$ von dem Wundrand ein und $\mathbf{I} \mathrm{cm}$ in die Tiefe; bei tiefer und langer Wunde aber müssen sie möglichst tief greifen, um die Bildung eines Hämatoms, Abszesses, Gangräne usw. zu vermeiden.

Die Nähte werden von mir immer nur so stark angezogen, daß die Wundränder gut koaptieren.

Die Pneumopexie halte ich für unnütz und irrationell, da sie zum Ausgangspunkt der Pleuraverwachsungen werden kann, die wir unbedingt vermeiden müssen.

Dic Tamponade der großen Höhle endlich halte ich für unnütz, unzureichend und infektionsgefährlich.

Aus diesem Grunde glaube ich, daß cin Eingriff an der Lungeohne eine breite Eröffnungkeinen Erfolgzeitigen könnte. 
Von kapitaler Wichtigkeit ist endlich die Nachbehandlung. Kampferöl, Chinin, physiologische Kochsalzlösung haben mir die besten Resultate gegeben.

Nach dem zweiten Tag hebe ich, wenn Lungenerscheinungen auftreten, den Kranken vorsichtig auf und untersuche ihn, um nicht durch eine unerwartete Pneumonie oder einen Hämothorax überrascht zu werden.

Dic größte Sorgfalt ist auf jeden Verbandwechsel zu verwenden, namentlich bei tamponierten Wunden. Die, Lunger.naht wird in der Mehrheit der Fälle, außer bei multiplen und allzu schweren Läsionen, Erfolg haben, wenn die Technik cine sorgfältige gewesen ist und eine rigoröse Asepsis durchreführt worden ist.

Zum Schlu: 3 fasse ich die sechs von mir operierten Fälle zusammen:

I. B e obachtung. - M. D., rgjähr. junger Mann, Schußwunde in der Herzgegend, Verletzung der Lunge, innere Blutung, Hämothorax, Puls 120. schwach.

Dringlicher Eingriff am 7. V. 1907. Thorakoplastischer Lappen, stark blutende Lungenwunde auf der Innenfläche nahe am vorderen Rande.

Die Ränder des Ein- und Ausschusses sind unregelmäßig, weshalb ich sie excidierte. Den Schußkanal wischte ich durch Durchziehen eines sterilisierten Haarseiles aus und vernähte die Öffnungen mit Katgut Nr. o. Toilette der Pleura, die ca. $600 \mathrm{~g}$ flüssiges Blut ent. hielt. Hermetischer Verschlub der Pleura, ke in e I) $\mathrm{ra}$ in a ge.

Injektion von physiologischer Kochsalzlösung. Normaler Verlauf.

Am 8. Tag Wunde vernarbt per primam intentionem. Am 20. Tag verläßt Pat. das Krankenhaus vollkommen geheilt.

2. B e ob achtung. -- G. S., 23jähr. Mann. Messerstich in der präkordialen Region; schwere innere Blutung. Ich diagnostizierte eine Verletzung der Lunge und möglicherweise des Herzens.

Dringlicher Eingriff : Breite Thorakotomic. Wunde an der Innenfläche beim Hilus. Starke Blutung, ca. I $1 / 2$ I.iter. Naht der Wunde mit Katgut, Toilette der Pleura, VerschluB ohne Draina ge.

Pat. genas whne Zwischenfall nach 20 Tagen. Es war eine oberflächliche Eiterung der Wunde an der oberen Partic eingetreten.

3. B e ob a chtung.- J. S., 22jähr. Mann. Thorako-abdominale Schußwunde, Verletzung der rechten Lunge, des Zwerchfells und der Leber. Innere Blutung, dringlicher Eingriff. Pat. ist ausgeblutet, Pul's I 40; Schweiß, starker Durst. Breite Thorakotomie unterhalb der rechten Brustwarze; ca. $500 \mathrm{~g}$ Blut in der Pleura. Der vordere äußre 
Rand des Unterlappens ist in der Ausdehnung eines Fünffrankenstückes zerfetzt. Massenligatur dieser Portion und Excision. Nach der Toilette der Pleura kam weiter Blut aus dem Abdomen durch das Loch in dem $Z$ werchfell. Seitliche Laparotomie. Transversale Incision längs des Rippenrandes, um eine blutende Wunde an der konvexen Fläche der Leber vernähen zu können. Starke abdominale Blutung.

An der unteren Fläche dauerte die Blutung fort; da nicht genäht werden konnte, tamponicrte ich. In diesem Augenblick Synkope, Puls unfühlbar, Pupillen erweitert. Endovenöse Injektion von 2000 g physiologischer Kochsalzlösung. Pat. kam wieder zu sich, ich nähte und verschloß die Wunden. Drainage unter die Leber, die Blutung stand. Nach 20 Tagen verläßt Pat. das Bett und bedarf noch einiger Pflege. Kein infektiöser Zufall.

Es ist dies ein Fall, wo ich das Maximum operativen Traumatismus an einem Individuum in extremis gesetzt habe. Die $\Lambda$ sepsis hat dabei eine sehr wichtige Rolle gespielt.

4. B e o b a ch t ung. - E. V., 28jähr. Frau. Thorako-abciominale Schußwunde, Verletzung der Lunge, des Zwerchfells und der Leber.

Die Lungenwunde sitzt in dem costo diaphragmalen Züngchen. Naht mit Katgut Nr. o, die Blutung stcht; Toilette der Pleura. Durch die Zwerchfellwunde tritt bei jeder Inspiration eine Menge Blut aus der Bauchhöhle in die Pleura. Laparotomie, Incision des Zwerchfells nach Resektion der letzten Rippen. Unregelmäßigge Wunde des linken Leberlappens, dic vernäht wird; die Blutung steht; Toilettc der Bauchhöhle; die benachbarten Eingeweide sind nicht verletzt. Naht des Zwerchfells. Wiederherstellung der Brust-Bauchwand ohne Drainage. Weiterer Verlauf sehr gut; 'Temperatur $37^{\circ}$, I'ul's normal. Vereinigung per primam intentionem. Am I4. Tagg ist Pat. vollkommen geheilt.

5. Beobachtung. . Gh. V., I9jähr. Bursche; Messerstich (Selbstmord) in der Herzgegend. Verletzung des Perikards und der linken Herzkammer. Herznaht; Heilung.

Breiter Lappen mit der Basis nach innen: breite Eröffnung des durch flussiges und geronnenes IBlut gedehnten Herzbeutel; ; die Herzbeutelwunde diente als Ventil; die C̈berfülle floß in die Pleura. Das Blut sprang aus dem Herzen in weitem Strahl. Ich nahm das Herz in die Handfläche, zogr es in die Operationswunde, drückte den Daumen auf die Wunde und vernähte sie dirch zwei Knopfnïhte mit Katgut Nr. o. Vollkommene Blutstillung. Toilette der Pleura und des I'erikards. Knopfnähte ins Perikard. Es blieben einige klaffende P'unkte im Perikard. Verschluß der Pleura; Naht des Lappens ohne I)rainage. Heilung nach zwei Wochen. Jie drei ersten Tage präkordiale Schmerzen, Atembeschwerden, häufiger I'uls, Temperatur $37,5-38,6^{\prime}$, die in drei Tagen lytisch abfiel. Leichter Hämothorax; Thorakozentese am 7. Tag ( $100 \mathrm{~g}$ serös blutiger Fliussigkeit).

Wenn ich diesen Fall auffuihre, so geschicht es, um nochmals die 
Harmlosigkeit der breiten Eröffnung der Pleura und des Verschlusses ohne Drainage darzutun.

6. B e ob a chtung. -.. A. T., zijähr. Mann. Schußwunde der Lunge. Naht ohne Drainage; breiter Lappen, Heilung ohne Zwischenfall von Bedeutung; vorübergehende Lungenerscheinungen. Heilung nach 25 Tagen.

7. Beobachtung. I). G., 2ojähr. Mann. Penetrierende Schu\&wunde. Durch ausgedehnte Pleuraverwachsungen zerfetzte Lungenwunde. Wiederholtes starkes Blutspeien. Die eröffneten Bronchien ließen die Luft austreten, die bei jeder Inspiration infolge der Blutung einen Blutschaum in der Wunde erzeugte. Kein Blut im Thorax, da die Pleurahöhle um die. Wunde herum nicht vorhanden war. Da ich keine Nabt anlegen konnte, tamponierte ich mit sterilisierter Gaze. Vernähung des Lappens um den Tampon. Pat. hustete während 3 Tage noch koaguliertes Blut in Form von Bronchialpfropfen aus.

Ich selbst habe den Verband unter der strengsten A sepsis gewechselt. Eine Woche lang floß eine scrös-schleimigeitrige Flüssigkeit der Bronchien ohne jede lokale oder allgemeine Reaktion ab. Pat. verlieB einen Monat nach dem Unfall das Bett. 